\title{
Verification of drag-reduction capabilities of stiff compliant coatings in air flow at moderate speeds
}

\author{
Andrey V. Boiko ${ }^{1}$, Victor M. Kulik ${ }^{2}$, Ho-Hwan Chun $^{3}$ and Inwon Lee ${ }^{3}$ \\ ${ }^{1}$ Institute of Theoretical and Applied Mechanics (ITAM), Siberian Branch of the Russian Academy of Science, Russia \\ ${ }^{2}$ Institute of Thermophysics, Siberian Branch of the Russian Academy of Science, Russia \\ ${ }^{3}$ Global Core Research Center for Ships and Offshore Plants (GCRC-SOP),Pusan National University, Korea
}

\begin{abstract}
Skin frictional drag reduction efficiency of "stiff" compliant coating was investigated in a wind tunnel experiment. Flat plate compliant coating inserts were installed in a wind tunnel and the measurements of skin frictional drag and velocity field were carried out. The compliant coatings with varying viscoelastic properties had been prepared using different composition. In order to optimize the coating thickness, the most important design parameter, the dynamic viscoelastic properties had been determined experimentally. The aging of the materials (variation of their properties) during half a year was documented as well. A design procedure proposed by Kulik et al. (2008) was applied to get an optimal value for the coating thickness. Along with the drag measurement using the strain balance, velocity and pressure were measured for different coatings. The compliant coatings with the thickness $h=7 \mathrm{~mm}$ achieved $4 \sim 5 \%$ drag reduction within a velocity range $30 \sim 40 \mathrm{~m} / \mathrm{s}$. The drag reduction mechanism of the attenuation of turbulence velocity fluctuations due to the compliant coating was demonstrated. It is envisioned that larger drag reduction effect is obtainable at higher flow velocities for high speed trains and subsonic aircrafts.
\end{abstract}

KEY WORDS: Compliant coating; Drag reduction; Wind tunnel experiment; Turbulent boundary layer.

\section{INTRODUCTION}

Verification of drag reduction capabilities of viscoelastic compliant coatings has remained the as subject of active research and debate. The phenomenal drag reduction as much as $60 \%$ by Kramer $(1957,1962)$ has never been reproduced afterwards. Those attempts were reviewed by Bushnell et al. (1977) and Gad-el-Hak (1996), who focused on the experiments employing the "soft" coatings, i.e., either porous material covered by thin film or gel-like substance. Those coatings are soft enough to form $\lambda$-shaped wrinkles on the surface and scarcely associated with drag reduction (Gad-elHak et al. 1984). Nevertheless, the laminar-turbulent transition delay, which was substantiated theoretically, was achieved in the experiments employing the soft compliant coatings (see, e.g., Carpenter 1990; Carpenter et al. 1985).

However, "stiff" rather than "soft" compliant coating consisting of a single viscoelastic layer is probably of primary practical importance for drag reduction applications. The stiff coatings are characterized by relatively large modulus of elasticity (usually more than about $0.5 \mathrm{MPa}$ ), which prevents the formation of surface wrinkles and

Corresponding author: Inwon Lee

e-mail:inwon@pusan.ac.kr increases coating durability. More precisely, a compliant coating is considered to be stiff if it remains hydraulically smooth under the influence of a flow.

The stiff compliant coating was introduced relatively recently; Kulik et al. (1991) reported about 20\% drag reduction with a model towed in a natural water basin at velocities $10-20 \mathrm{~m} / \mathrm{s}$. The same coating was subsequently tested in a cavitation tunnel by Choi et al. (1997) and found to be result in drag reduction of about $7 \%$ at velocities $1 \sim 5$ $\mathrm{m} / \mathrm{s}$. In addition, the fluctuations of wall pressure and wall shear stress as well as the turbulence intensity were found to be attenuated in the compliant coating case. It is worthwhile to mention that two experiments were carried out at different flow velocities and in a considerable time span. Later Bandyopadhyay et al. (2005) observed that the drag reduction effects of the similar compliant coatings almost vanished after a long periods of times such as 200 days. This was attributed to the aging of the viscoelastic material, which is the slow transformation from rubber to resin due to the progress of cross-linking reaction.

The main difficulty for the testing and application of the compliant coatings in air flows is that the significant difference between the densities of the coating and the fluid causes the attenuation of the interaction of the coating with the turbulent pressure fluctuations. This should lead to a reduced, if any, drag reduction efficiency of the coatings 
particularly at small flow velocities and, consequently, to more strict requirements for experiment accuracy. Hence, most of the related studies in air up to now were devoted to the control of laminar-turbulent transition by means of the soft coatings (Huang and Johnson 2007). A notable exception to this trend is the wind tunnel experiment of Kornilov et al. (2004), who tested a quite stiff viscoelastic coating $(E=0.75$ $M P a$ ) consisted of a monolithic layer of heat-resistant lowtemperature synthetic rubber. In this experiment, the maximum $5 \%$ of drag reduction was achieved in the velocities $10 \sim 15 \mathrm{~m} / \mathrm{s}$.

While most existing literature on compliant coatings deals with the detailed description regarding the flow measurement, they lack information on the mechanical properties of the coating which could allow one to predict the drag reduction efficiency and the appropriate flow velocity range based on some theoretical approaches. Sometimes, the mechanical properties of the compliant coating need be defined under the action of dynamic loads, because the coating will undergo deformation by the turbulent pressure fluctuations. To the knowledge of the authors, very little study took the dynamic properties of coating material into considerations.

Recently, a novel measurement technique for the viscoelastic dynamic properties of compliant coating has been proposed by the authors (Kulik et al. 2009). Based on the standard vibration measurement instruments, this method facilitates the measurement of the modulus of elasticity (or a shear modulus) and the loss tangent in a wide frequency range of $10 \sim 4,000 \mathrm{~Hz}$ at relative deformations from $10^{-4} \%$ to $5 \%$. That frequency range is of particular significance in turbulent flow applications. Extension of this method enabled the experimental determination of the dynamic Poisson's ratio, as described in Boiko et al. (2010). In Kulik et al. (2008), empirical formulae between the oscillating characteristics (resonant frequency, propagation velocity of deformation wave) and the viscoelastic material properties were derived from the analysis of two-dimensional deformation wave in a compliant coating. These formulae were then combined with the drag reduction condition of Semenov (1991) to yield flow velocity range where drag reduction could be expected.

The present study is aimed at the experimental verification of the hypotheses in Kulik et al. (2008). Flat plate compliant coating inserts were installed in a wind tunnel and the measurements of skin frictional drag and velocity field were carried out. The compliant coatings with varying viscoelastic properties had been prepared using different composition. In order to optimize the coating thickness, the most important design parameter, the dynamic viscoelastic properties had been determined experimentally. The structure of the present paper is as follows; in the following section, the sample preparation procedures are described in detail. Section 3 deals with the optimization process of the coating. Finally, some results on turbulent boundary layer in a wind tunnel and comparison of the experimentally estimated skin friction modifications with the predictions are presented in sections 4 and 5.
DYNAMIC VISCOELASTIC PROPERTIES OF THE COMPLIANT COATING MATERIAL

A silicon RTV (Room Temperature Vulcanizing) rubber (Dow Corning Silastic $S-2^{\circledR}$ ) was employed to prepare for the coatings. This is a low-molecular PDMS (polydimethylsiloxane) with the structural formula [-O$\left.\mathrm{Si}\left(\mathrm{CH}_{3}\right)_{2}-\right]_{\mathrm{n}}$ which is initially (before being mixed with the cross linking catalyst) a very viscous liquid with the viscosity of 90 Poise. The standard composition recommended by the manufacturer consists of $90 \%$ of the PDMS resin fluid and $10 \%$ of the cross linker, which will be denoted as $\mathrm{C} 10.0$ composition hereinafter. In order to test materials with diversified viscoelastic properties, the standard composition was slightly varied to get C12.5 composition (87.5\% resin + $12.5 \%$ cross linking catalyst) and C7.5 composition (92.5\% resin $+7.5 \%$ cross linking catalyst). Care was taken to ensure the removal of any minute air bubbles by keeping both substances at $-0.15 \mathrm{~atm}$ gauge pressure for two hours. The resin fluid and the catalyst were then mixed slowly by a rotating stirrer during 20 minutes. The mixture was again degassed in the vacuum pressure for about 1 hour. Then, the mixture was poured with a plunger both in cylindrical moulds and plate mould. The former is for the viscoelastic property measurement and the latter for the wind tunnel test. Thus, the samples for the viscoelastic property measurement were manufactured from the same substance as the wind tunnel test.

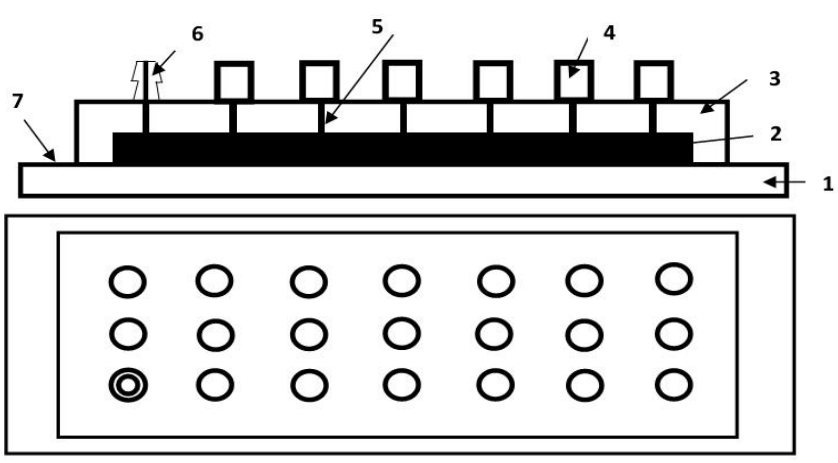

(a) Schematic diagram of the mould to prepare for the compliant coating; 1:base plate, 2:compliant coating, 3:removable insert, 4:dilators (hollow cylinders), 5:holes, 6: connecting pipe, 7 : film.

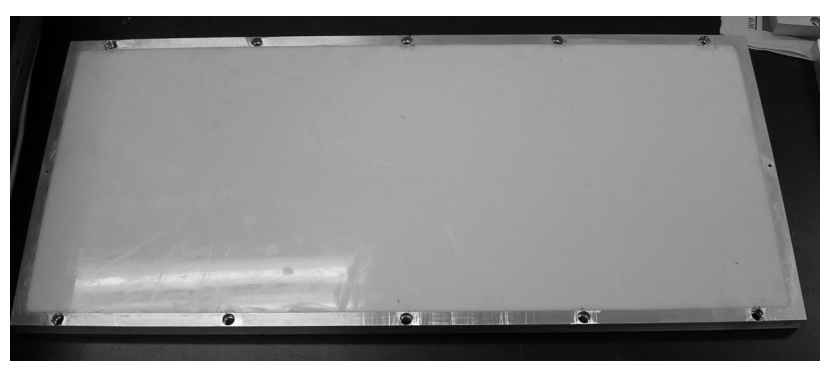

(b) Photo of a typical compliant coating test plate.

Fig.1 Compliant coating for the wind tunnel test. 
The mould to prepare for the wind tunnel test coatings is illustrated in Fig. 1 (a). The PDMS mixture is poured into the space (2) with sizes $530 \times 230 \times h \mathrm{~mm}^{3}(h=1-7 \mathrm{~mm})$ between the base plate and the removable insert (3). The mixture liquid is made to fill up the space (2) and the channels (5) to reach the middle of the dilator pipes (6), thereby preventing for the formation of the cavities inside the coating due to the contraction during the curing process. To provide a sufficient adhesion the mould surface was washed by a primer 24 hours before the coating manufacturing. Flexible acrylic plate with thickness about $1 \mathrm{~mm}$ was placed between the base plate (1) and the removable insert (3) to provide the smoothness of the outer coating surface and simplify the coating removing. The compliant coating (2) and the removable insert (3) form a wind tunnel test plate, as shown in Fig. 1 (b).

The technique of determining the dynamic viscoelastic properties is described in detail in Kulik et al. (2009) and Boiko et al. (2010). The measurements were performed in the linear deformation region at relative deformation of about $\sim 10^{-3} \%$, which corresponds to the expected magnitude of the coating deformation in a turbulent flow. In Fig. 2, it is shown that the material deforms linearly in the frequency range under consideration up to the relative deformation $\varepsilon$ of about $1 \%$.
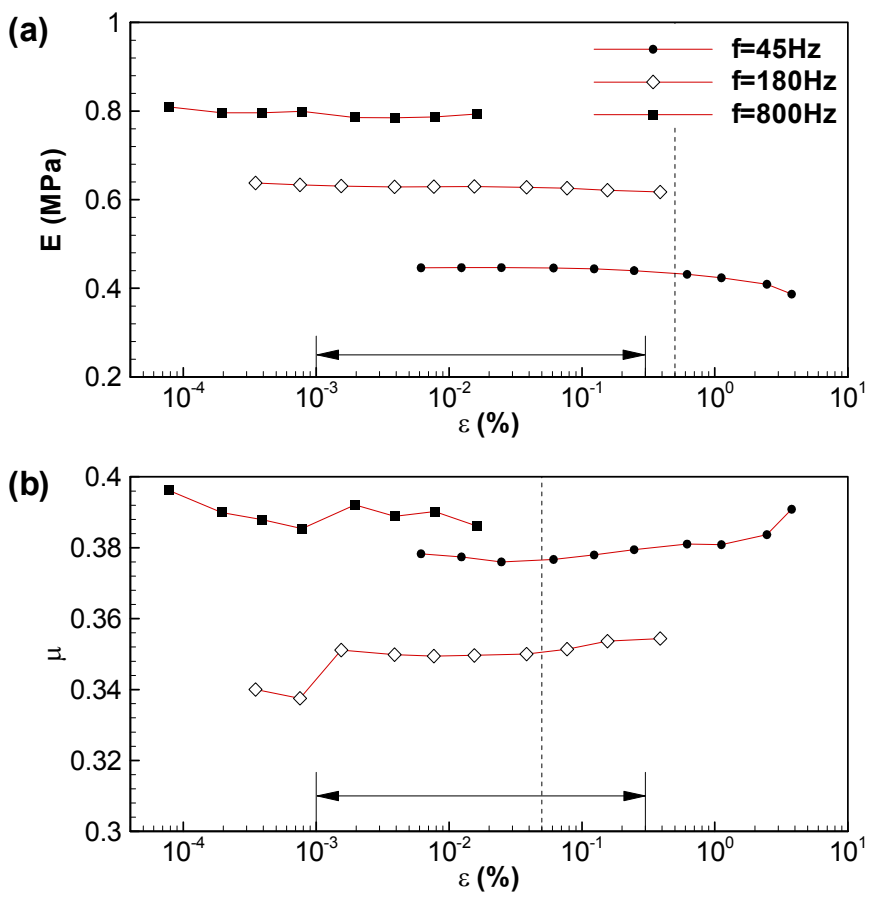

Fig. 2 Dependence of modulus of elasticity $E$ and loss tangent $\mu$ on the relative deformation $\varepsilon$ for $\mathrm{C} 7.5$ composition.

Figure 3 plots the frequency dependence of the modulus of elasticity $E$ and the loss tangent $\mu$ for three compositions under consideration which was measured 15 days after the sample preparation. The aging characteristics of the materials over half a year are presented in Fig. 4 for two representative frequencies. At other frequencies in the measured frequency range, the viscoelastic properties exhibited similar changes with time. As seen, after the first 40 days of pronounced changes, the values of the modulus of elasticity and the loss tangent become saturated. As all the samples were prepared one by one during one week, the subsequent wind tunnel tests were carried out 2 month after the sample preparation, when the properties became practically constant. The properties of the standard C10.0 composition are subject to the minimum changes with time among three tested compositions

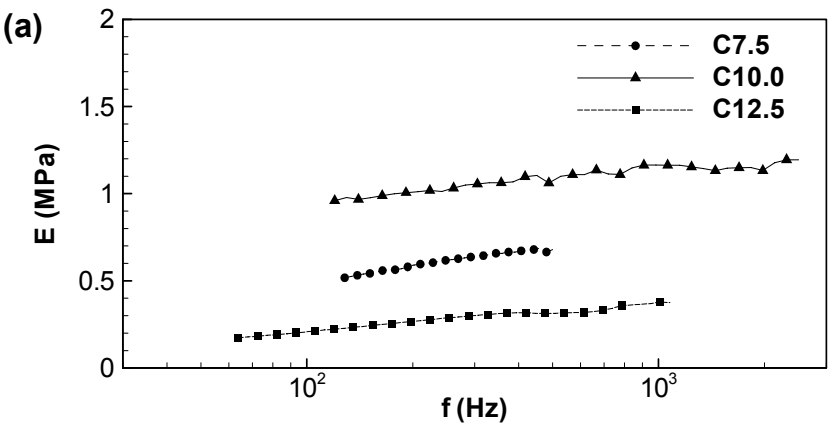

(b)

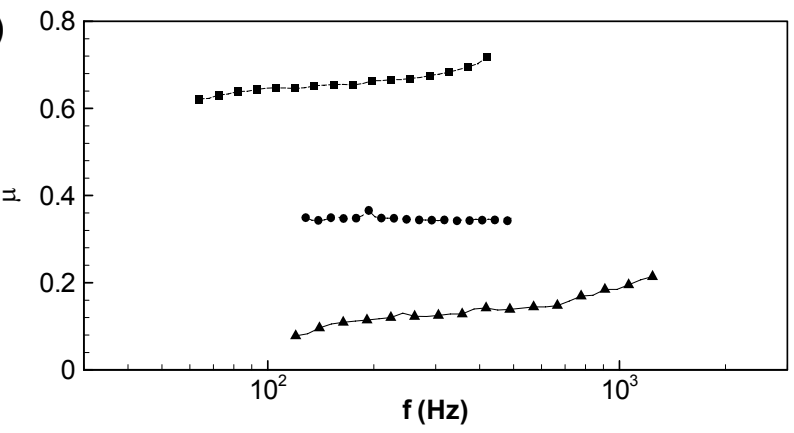

Fig. 3 Modulus of elasticity $E$ and loss tangent $\mu$ vs. frequency.

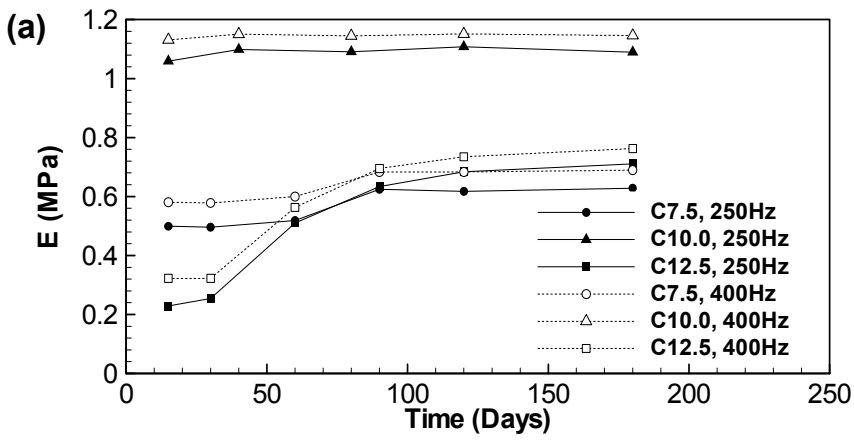

(b)

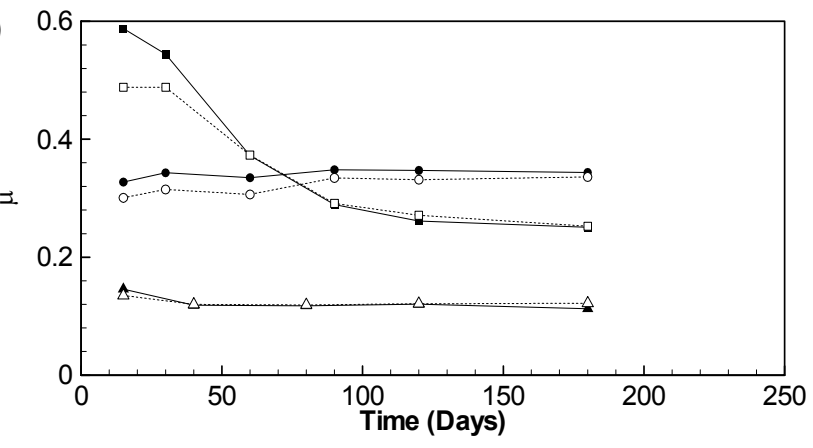

Fig. 4 Modulus of elasticity $E$ and loss tangent $\mu$ as functions of time. 
Also, the standard composition of $\mathrm{C} 10.0$ led to the largest modulus of elasticity and the smallest loss tangent among three tested compositions. The standard composition is then considered to be optimal in terms of polymerization process, thereby giving rise to stiffest material. The $\mathrm{C} 7.5$ composition is shown to be more stable than C12.5. Although the initially much reduced modulus of elasticity for the C12.5 composition would be preferential in terms of maximizing coating deformation, the unstable aging characteristics is evidently detrimental to the long-term stability of the coating. Therefore, the C12.5 composition was excluded in subsequent tests. As seen in Fig. 5, dynamic Poisson's ratio $\sigma$ appeared to be the same for both materials and practically constant and equal to $0.485 \pm 0.005$ in the studied frequency range.

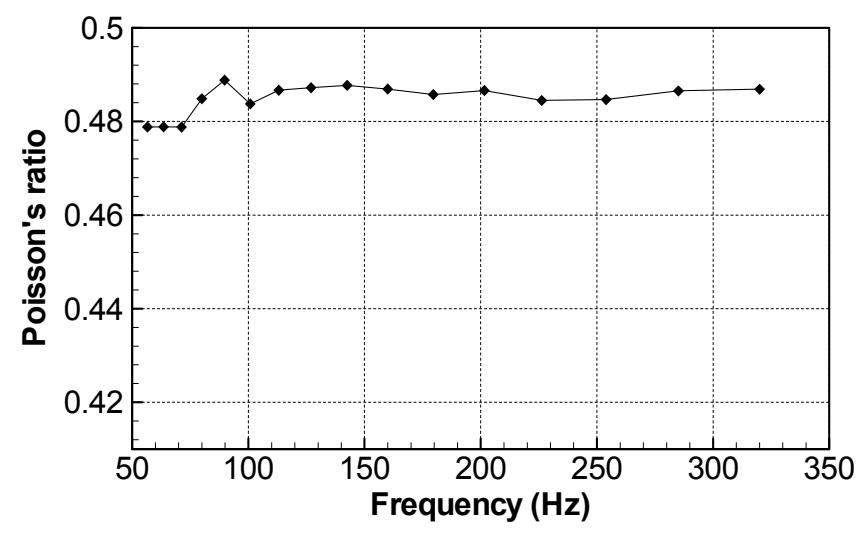

Fig. 5 Poisson's ratio $\sigma$ as a function of frequency.

\section{PREDICTION OF DRAG REDUCTION}

To the knowledge of authors, there is no proven reliable engineering method to predict the skin friction reduction effect by a compliant wall for turbulent flows. However, it is possible to indicate a range of parameters of the coating for a given flow velocity, for which one can expect the drag reduction.

The mechanism of possible drag reduction is explained by changes in generation of Reynolds stresses in the boundary layer above the 'stiff 'coating (Kulik 2011).

$$
\tau=\rho\left\langle\left(u_{\text {flow }}+u_{\text {coat }}^{\prime}\right)\left(v_{\text {flow }}+v_{\text {coat }}^{\prime}\right)\right\rangle,
$$

where $u_{\text {flow }}, v_{\text {flow }}$ are undisturbed velocity fluctuations in the streamwise and wall-normal directions; $u_{c o u t}^{\prime}, v_{\text {cout }}^{\prime}$ are the disturbances introduced by the compliant coating in the corresponding velocity components. The value and the sign of the changes in the Reynolds stresses depend both on the amplitude of the induced velocity disturbances and the phase shift between them.

The proposed method of optimizing the characteristics of the coating is based on the analyses of both its response to an external forcing (Duncan et al. 1985; Kulik et al. 2005a; Kulik et al. 2005b; Kulik et al. 2008) and the generation of turbulence at the compliant surface (Semenov 1996; Semenov 2009) and requires that

- the interaction between the flow and the coating is maximum.

- the resonant frequency of the coating is in a certain frequency range.

In contrast to the soft coatings, the amplitude of the surface deformation for a stiff coating is less than the thickness of the laminar sublayer; hence, the coating is always hydraulically smooth. However, in the frequency range of the coating and flow interaction (in the vicinity of the resonant frequency of the coating) the speed of its movement may be comparable with the turbulent velocity fluctuations near the wall (Kulik et al. 2005a; Kulik et al. 2005b).

Maximum interaction between the flow and the coating would be achieved when the following requirements, obvious from practical viewpoint, are fulfilled;

a) the convection velocity of near-wall vortical structures should be equal to the velocity of propagation of disturbances inside the coating, $U_{\text {conv }}=V_{\text {coat }}$.

b) the frequency of the forcing should be equal to the resonant frequency of the coating, $f=f_{0}$.

According to the two-dimensional model of deformation of viscoelastic coating (Kulik et al. 2008), the resonance frequency is determined by the formula

$$
\frac{\omega_{0} H}{C_{t}}=2.244+1.96 \sigma
$$

where $\quad C_{t}=\left[\frac{E}{2 \rho(1+\sigma)}\right]^{1 / 2}$

is the propagation velocity of a transverse shear wave, $\rho$ is the density of the material, $h$ is coating thickness. As $V_{\text {coat }}$ depends significantly on the frequency of the interaction (Gad-el-Hak et al. 1984; Duncan et al. 1985; Kulik et al. 2008), it is reasonable to select the $V_{\text {coat }}$ at the resonance frequency $f=f_{0}$ for the maximum interaction between the coating and the flow. According to Kulik et al. (2008), the wave propagation velocity at the resonance frequency is given as

$$
\frac{V_{0}}{C_{t}}=2.826-4.5 \sigma+3.9 \sigma^{2}
$$

Analysis of turbulence-energy generation by Semenov (1991) indicated that the generation of turbulence above the compliant wall is reduced if the nondimensional resonance frequency of the coating is in the range

$6.67 \times 10^{-3}<\frac{f_{0} v}{u_{\tau}^{2}}<2.00 \times 10^{-2}$ 
where $v$ and $\mathrm{u}_{\tau}$ are the kinematic viscosity and the friction velocity, respectively. It was pointed out by Choi et al. (1997) that this condition suggests that the coating response contributes to weakening the upwash movement during the ejection event. Meanwhile, it is notable that the frequency range in Eq. (3) corresponds approximately to the energycarrying part of the spectra of the turbulent pressure fluctuations.

On the other hand, Kulik et al. (2005a) concluded that the coating deformation is maximized when the wavelength of pressure fluctuations convecting with velocity $U_{c}$ is equal to the wavelength of surface. Hence, a drag reduction would be expected when an optimal interaction of the coating with the flow occurs with the following conditions

- temporal factor : coincidence of the resonant frequencies in Eq. (3)

- spatial factor : coincidence of the wavelengths

$V_{0}=U_{C}=0.7 \sim 0.9 U_{0}$

For a material with given viscoelastic properties $E, \mu$ and $\sigma$ and at a given flow velocity $U_{0}$, these conditions make it possible to determine the optimal thickness $h$ of the coating. The conditions, as seen, are necessary, rather than sufficient for the drag reduction. In the series of studies by Amphilokhiev et al. (2000), a large influence of the level of infow turbulence on the drag reduction was pointed out. Probably, this is related to the requirement that the pressure fluctuations of the turbulent flow should have certain coherence (Kulik et al. 2005b). Otherwise, the compliant coating has little time to interact with the applied forcing and the surface deformation becomes negligible. In addition, another significant parameter for the description of dynamic deformation is the loss tangent $\mu$ of the coating material. For $\mu<0.1$, a lot of periods of oscillations are required to reach the desired level of deformations. For $\mu>0.4$ only one period is enough, but the steady-state amplitude of deformations will be much smaller than that for small value of loss tangent (Kulik et al. 2005b).

Let us consider the optimization process of the coating thickness. It is noteworthy that the two conditions in Eqs. (3) and (4) are expressed as inequalities in the flow velocity $U_{0}$ at a fixed value of coating thickness. Table 1 presents the results of such a sample calculation of the flow velocity range in which the optimal interaction of the coating with the flow and the drag reduction are expected. Here, the thickness of the coating $h=3 \mathrm{~mm}$ is assumed. In these calculations, the viscoelastic material properties were taken from the dynamic property measurements described in section 2. Although the samples for those measurement were in cylindrical shape, i.e., different from the planar compliant coating in the wind tunnel test, this causes no problem because the influence of geometry had been eliminated from the two-dimensional deformation analysis employed in this study. It is also supposed that the aging characteristics of the cylindrical samples and the plane coating are the same, i.e. the aging is independent on the shape and the size of a sample. The density and the Poisson's ratio were all set to same values of $\rho=1.13 \times 10^{3} \mathrm{~kg} / \mathrm{m}^{3}$ and $\sigma=0.485$ for all coatings considered here.

As seen, no coating in Table 1 satisfies both requirements, i.e., there is little overlapped velocity ranges from the temporal factor and the spatial factor. For instance, for the C7.5 composition, the deformation wavelength at the resonant frequency coincides with the convective wavelength of the pressure fluctuations for the flow speed of $U_{0}=25 \mathrm{~m} / \mathrm{s}$. However, the resonant frequency in this case is out of the major frequency range of the turbulent pressure fluctuations. Similarly, two other cases listed in Table 1 do not lead to the overlap. A closer inspection of Table 1 indicates that the standard C10.0 case would be the most promising in that there is slight overlap between the upper bound of the spatial factor and at the lower bound of the temporal factor.

In order to widen the overlapped velocity range, there are two options; either increases the upper bound from the spatial factor or decrease the lower bound from the temporal factor. Combining Eq. (2) with Eq. (4) and evaluating with $\sigma=$ 0.485 expresses the spatial factor in the form of the velocity inequality

$1.73\left[\frac{E}{2 \rho(1+\sigma)}\right]^{1 / 2}<U_{0}<2.23\left[\frac{E}{2 \rho(1+\sigma)}\right]^{1 / 2}$.

The upper bound is independent of the thickness. Therefore, it is needed to enlarge the modulus of elasticity or to reduce the density and the Poisson's ratio, which is hardly possible once the material has been decided. Another option would be to decrease the lower bound of the temporal factor which becomes

$$
2.95<\frac{C_{t}}{U_{0}^{1.8} h}<8.90
$$

in the present study. The lower bound could be easily reduced by increasing the coating thickness. From this finding, the coating thicknesses were varied from the initial value $h=3 \mathrm{~mm}$ to obtain wider overlapping region in the flow velocity for each composition. Exemplary results are listed in Table 2, where the overlapping condition is shown $t$ be satisfied. The adjusted coating thicknesses $h=6 \mathrm{~mm}$ for $\mathrm{C} 10.0$ and $h=8 \mathrm{~mm}$ C7.5 compositions were then taken into consideration in designing the mold to manufacture the wind tunnel test coating (see Fig. 1). As a matter of fact, the average value of $h=7 \mathrm{~mm}$ was selected for the sake of convenience.

Table 1 Expected effective flow velocities for $h=3 \mathrm{~mm}$.

\begin{tabular}{|c|c|c|c|c|c|}
\hline Material & $\begin{array}{c}E, \\
M P a\end{array}$ & $\begin{array}{c}f_{0,} \\
k H z\end{array}$ & $\begin{array}{c}V_{0}, \\
m / s\end{array}$ & $\begin{array}{c}\text { Spatial } \\
\text { factor } \\
U_{0}, m / s\end{array}$ & $\begin{array}{c}\text { Temporal } \\
\text { factor } \\
U_{0}, \mathrm{~m} / \mathrm{s}\end{array}$ \\
\hline Standard & 1.15 & 3.08 & 28.62 & $31.8-0.9$ & $40.6-72.8$ \\
\hline $\mathrm{C} 7.5$ & 0.60 & 2.22 & 20.66 & $23.0-9.5$ & $32.8-60.7$ \\
\hline $\mathrm{C} 12.5$ & 0.30 & 1.57 & 14.60 & $16.2-0.9$ & $27.0-50.0$ \\
\hline
\end{tabular}


Table 2 Estimation of optimal thickness of the compliant coatings.

\begin{tabular}{|c|c|c|c|c|c|}
\hline Material & $\begin{array}{c}h, \\
m m\end{array}$ & $\begin{array}{c}E, \\
M P a\end{array}$ & $\begin{array}{c}f_{0,} \\
H z\end{array}$ & $\begin{array}{c}\text { Spatial } \\
\text { factor } \\
U_{0,} m / s\end{array}$ & $\begin{array}{c}\text { Temporal } \\
\text { factor } \\
U_{0,}, \mathrm{~m} / \mathrm{s}\end{array}$ \\
\hline Standard & 6 & 1.15 & 1540 & $31.8-40.9$ & $26.7-49.3$ \\
\hline $\mathrm{C} 7.5$ & 8 & 0.60 & 833 & $23.0-29.5$ & $19.1-35.1$ \\
\hline $\mathrm{C} 12.5$ & 10 & 0.30 & 471 & $16.2-20.9$ & $13.9-25.6$ \\
\hline
\end{tabular}

\section{WIND TUNNEL EXPERIMENTAL SETUP}

The experiments were carried out in the wind tunnel of Aerospace Department of Pusan National University. The tunnel test section is $2 \mathrm{~m}$ long with $0.7 \times 0.7 \mathrm{~m}^{2}$ cross section. The schematic diagram of the wind tunnel, along with the photograph of the test section during flow measurement by hot wire anemometry, is presented in Fig. 6.
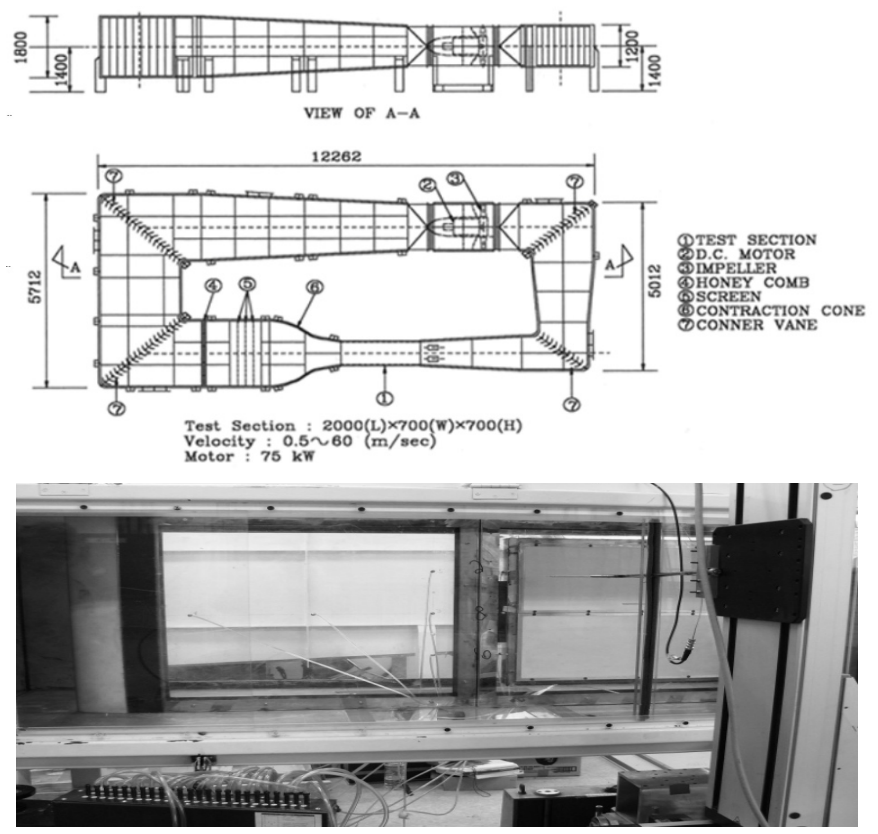

Fig. 6 Schematic diagram of the wind tunnel and photo of the test section.

A flat plate was mounted vertically in the central part of the test section. The plate is $80 \mathrm{~mm}$ thick and consists of four parts as shown in Fig. 7. The first (2) and the second (3) square sections served to mount the inserts with the compliant (5) and reference (6) coatings are interchangeable. The leading edge (1) has the elliptic cross section with the axis ratio $3: 1$. The trailing edge flap (4) could be deflected to adjust pressure gradient near the leading edge. It is known that the constant temperature anemometer (CTA) data could be affected by heat-conduction effects in the near-wall region. In order to minimize such effect, a $1 \mathrm{~mm}$-thick $\mathrm{C} 10.0$ coating instead of a metal rigid plate was used as the reference one to provide the same heat-conductivity. This coating causes negligible flow modification compared with the rigid plate. The coordinate system is also shown in Fig. 7. The origin of the coordinate system is at the point of intersection of the plate leading edge and its centerline. The streamwise coordinate is denoted as $x$, wall-normal coordinate as $y$ and spanwise coordinate as $z$.

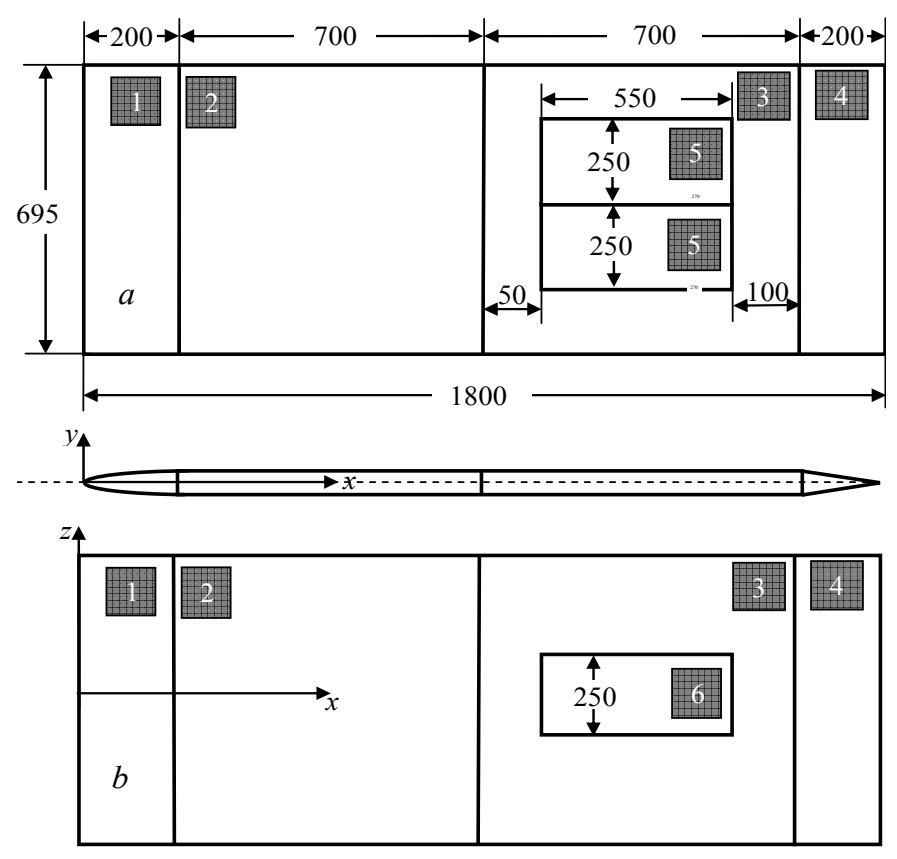

Fig. 7 Flat plate setups for different measurements; (b) drag balance measurement, 1:leading edge, 2:first section, 3 :second section, 4 :trailing edge flap, 5:insert with two compliant coating, 6:insert mounted on a strain-gage balance.

Artificial tripping of the boundary layer was provided by a rod with a diameter of $1.5 \mathrm{~mm}$, mounted over the plate span at a distance of $160 \mathrm{~mm}$ from the leading edge. Additionally a $45 \mathrm{~mm}$ wide strip of a large-grit sandpaper (with smoothed edges) was located right downstream of the rod.

In the first series of the measurements, boundary layer characteristics above the inserts were obtained. For direct comparison of the characteristics between the reference and the test coating, both of them were mounted simultaneously as shown in Figs. 6 and 7 (a). Streamwise velocity was measured using a miniature I-type fiber-film probe (Dantec $55 R 01)$ connected to the constant temperature hot-wire anemometer (Dantec StreamLine). Fiber-film probes are less susceptible to particle contamination and, hence, show better long-term stability than classical wire probes. To provide even better accuracy of CTA data, an RTD (Resistance Temperature Detector) placed inside the wind tunnel test section was used together with StreamLine hardware to provide automatic temperature compensation during the measurements. The data were acquired for 15 seconds with $10 \mathrm{kHz}$ sampling frequency at every measurement point.

The fiber-film probe was calibrated against a Pitot-static tube in the freestream of the test section. The tube was connected to a differential pressure transducer (Omega 
PX2650-10D5V) with the dynamic range of 10 inch $\mathrm{H}_{2} \mathrm{O}$. A modified King's law formula for a single wire calibration recommended in Johansson and Alfredsson (1982)

$$
U=k_{1}\left(E-E_{0}\right)^{1 / n}+k_{2} \sqrt{E-E_{0}},
$$

where $E$ is the anemometer output voltage at the velocity $U$ and $E_{0}$ is the voltage at $U=0$, was used. The calibration function was found to fit the calibration points (after an automatic adjustment of constants $k_{l}, k_{2}$, and $\mathrm{n}$ with a specially designed computer program) over the wide velocity interval with relative errors less than $1 \%$.

In the second series of the measurements, the setup in Fig. 7 (b) was used. It was served to measure the drag of the insert with a compliant or reference coating. A strain-gage balance was placed inside the second section. The test coating was mounted flush with the plate surface with 0.2 to $0.5 \mathrm{~mm}$ clearance at the insert perimeter. The signals from the balance were amplified by a three channel bridge amplifier (Endevco Model 136). An accelerometer (Brüel \& Kjcer Type $4514 B-002)$ was mounted on the metal frame of the straingage balance. This sensor measured acceleration in the $y$ direction (normal to the plate). This sensor was connected to a four-channel Deltatron conditioning amplifier (Brüel \& Kjor Nexus Amplifier Type 2693A).

Figure 8 illustrates a general schematic of the experimental setup. The tests were performed at free stream velocities $U_{0}=20 \sim 42 \mathrm{~m} / \mathrm{s}$ measured above the inserts by a Pitot-static tube (2).

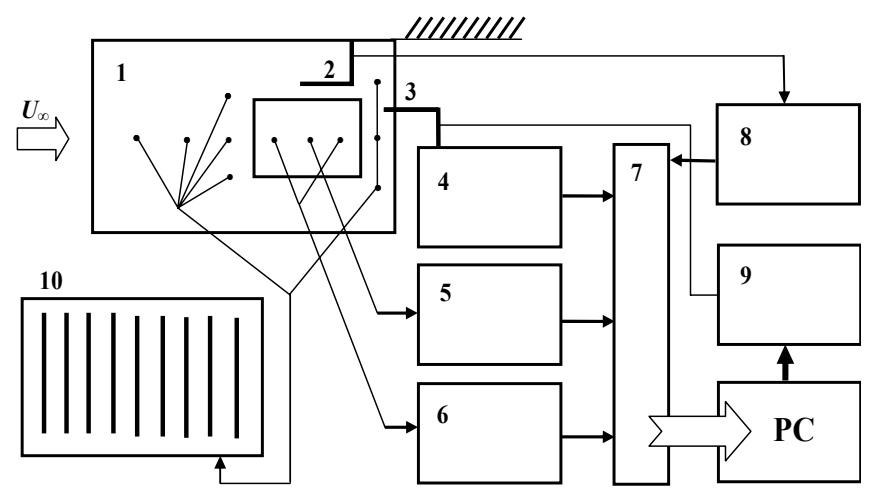

Fig. 8 Schematic diagram of instrumentation, 1:plate with insertion and pressure taps, 2:Pitot-static tube, 3:hot-wire probe, 4:constant temperature anemometer, 5:bridge amplifier, 6:Deltatron conditioning amplifier, 7:A/D converter, 8:pressure transducer, 9:traversing mechanism, 10: multiple-tube inclined differential manometer.

Static pressure gradient over the plate was controlled by 11 pressure taps of $0.5 \mathrm{~mm}$ diameter located along the centerline as well as along the span at upstream and downstream of the insert. The pressure distribution was measured by a multipletube differential alcohol micromanometer (10). Electrical signals from respective amplifiers $(4,5,6,8)$ were digitized an A/D converter (National Instrument PCI-6035E) (7) and logged into a personal computer. A PC controlled standard three-axes traversing mechanism (9) allowed positioning of the fiber-film probe (3) with $0.005 \mathrm{~mm}$ accuracy. The CTA measurements were performed at three downstream positions, namely 25,240 and $470 \mathrm{~mm}$ downstream of the leading edges of the insert. Nominally zero streamwise pressure gradient above the inserts was obtained by adjusting the plate and trailing edge flap (4) angle of attack.

\section{RESULTS OF THE WIND TUNNEL EXPERIMENT}

The main emphasis of this section to consider the efficiency of the compliant surfaces to modify the drag with respect to the reference surface. The measured level of vibrations of the plate in wall-normal direction is shown in Fig. 9.

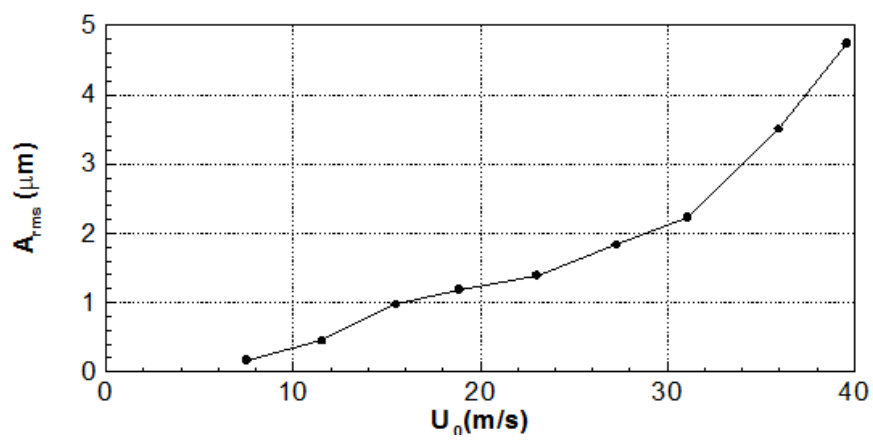

(a) dimensional amplitude

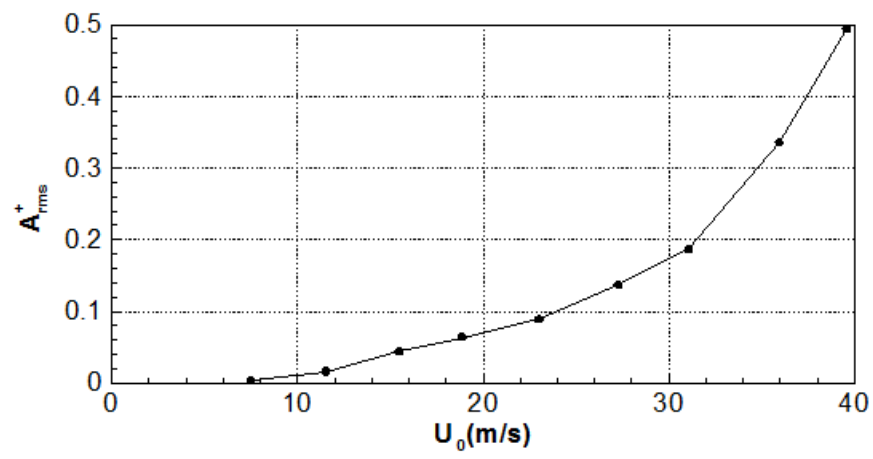

(b) nondimensional amplitude

Fig. 9 Vibrations of the flat plate at the midpoint of the strain-gage balance.

Recalculation of measured vibration acceleration to rootmean-square (rms) amplitude of displacement in wall-normal direction was performed using the formula:

$A_{\mathrm{rms}}=\sqrt{2 \sum \frac{\Phi_{\omega}^{2}}{\omega^{4}}}$,

where $\Phi_{\omega}$ is a spectral component of the vibration acceleration at a discrete frequency $\omega$. Then, the resulting value of $A_{r m s}$ was averaged over 200 realizations. Values of $\Phi_{\omega}$ were uobtained using discrete Fourier transform of the sampled data. Dimensionless amplitude of the vibrations is 
defined as $A^{+}=A_{r m s} u_{\tau} / v$. The friction velocity $u_{\tau}$ is estimated here roughly as $u_{\tau}=\left[0.03 U_{0}^{2}\left(\operatorname{Re}_{L}\right)^{-0.2}\right]^{0.5}$ (Schlichting 1968), where $L=1.225 \mathrm{~m}$ corresponds to the middle section of the insert. Even at the highest free stream velocity under study the amplitude of vibrations is less than $A_{\text {rms }}=5 \mu m$ (Fig. 9 (a)) or $A^{+}=0.5$ (Fig. 9 (b)). This indicates that the wall was hydrodynamically smooth in all cases.

To document flow characteristics, detailed measurements of mean velocity profiles $U(y)$ were carried out inside the boundary layer at several downstream locations along the centerline of the inserts. The profiles were used to estimate integral boundary layer characteristics and local skin friction. Analysis of the data indicates that the integral characteristics of the boundary layer correspond quite well to the physical concepts on properties of the flat-plate turbulent boundary layer at nominally zero pressure gradient. For example, the streamwise growth of the momentum thickness Reynolds number $\operatorname{Re}_{\theta}=U_{0} \theta / v$ is plotted against the streamwise Reynolds number $\operatorname{Re}_{x}=U_{0} x_{\text {eff }} / v$ in Fig. 10. Here, $x_{\text {eff }}$ denotes the downstream distance from an effective origin of the turbulent boundary layer. As seen, the experimental data shows quite good agreement with the empirical formula based on power-law velocity profile assumption $\operatorname{Re}_{\theta}=0.036 \operatorname{Re}_{x}^{0.8}$ by Schlichting (1968).

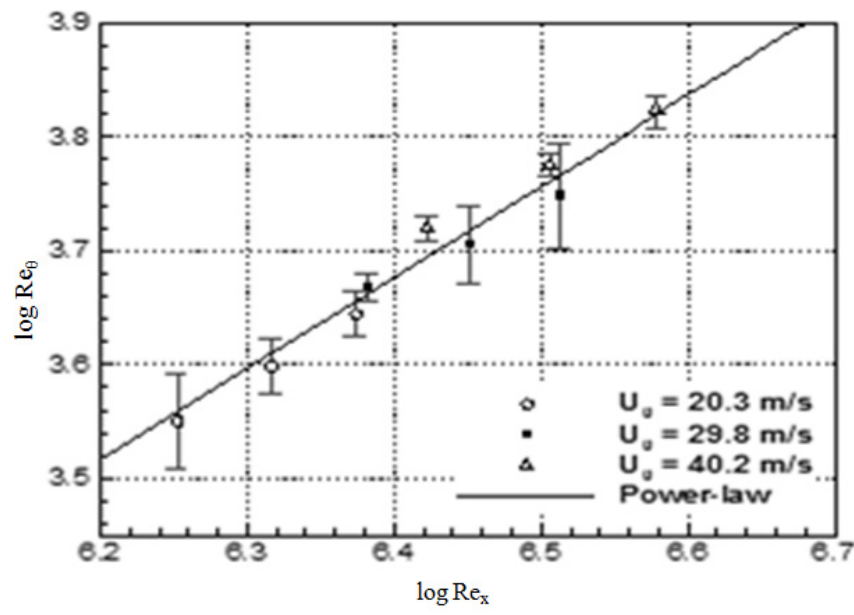

Fig. 10 Dependence of momentum thickness on Reynolds number.

Figure 11 demonstrates the velocity profiles in wall variables for $\left(u^{+}=u / u_{\tau}, y^{+}=y u_{\tau} / v, u_{\tau}=\sqrt{\tau_{w} / \rho}\right.$, where $\mathrm{u}$ is the streamwise velocity at distance y normal the wall) $U_{0}=29.8$ $\mathrm{m} / \mathrm{s}$. The canonical logarithmic velocity profile (straight solid line)

$u^{+}=\frac{1}{\kappa} \ln y^{+}+B$

with $\kappa=0.41$ and $B=5.0$ and the viscous sublayer profile $u^{+}$ $=y^{+}$(dashed curve) valid in the viscous sublayer are also given for comparison. Similarly as Fig. 11, most of the velocity profiles are found to be in close agreement with the canonical logarithmic profile, which supports the establishment of equilibrium turbulent boundary layer in the region of measurements. The discrepancy between the viscous sublayer profile $u^{+}=y^{+}$and the measured profiles is quite usual for hot-wire measurements, indicating the pronounced heat-conduction effects in the vicinity of the wall.

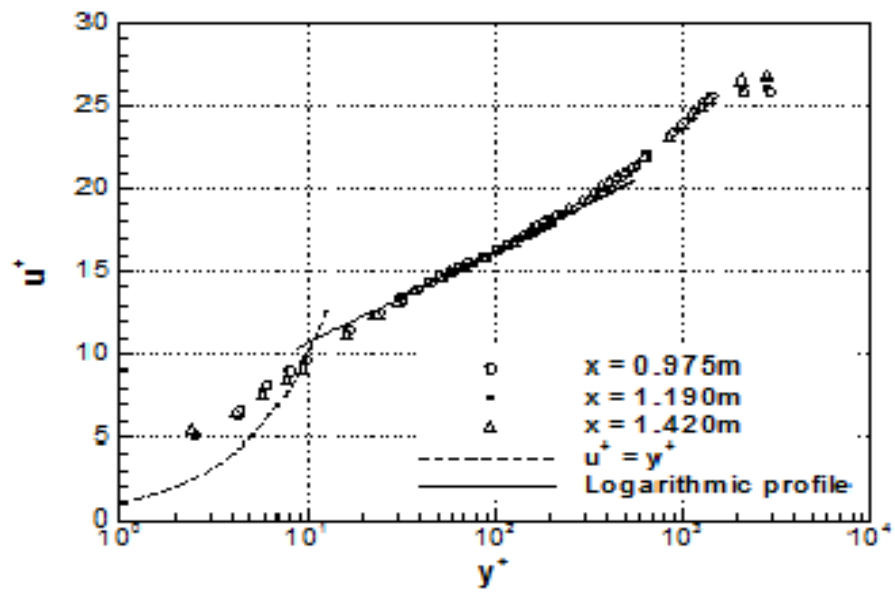

Fig. 11 Mean velocity profiles in wall variables for the reference coating at $U_{0}=29.8 \mathrm{~m} / \mathrm{s}, x=0.975(\circ), 1.190(\bullet)$, $1.420 \mathrm{~m}(\Delta)$; viscous sublayer profile $u^{+}=y^{+}$(dashed curve), logarithmic profile (solid line).

The validity of the measurements performed for the reference surface can be further illustrated by dependence $C_{f}=f\left(R e_{\text {xeff }}\right)$ plotted in Fig. 12. The measured values of $C_{f}$ were estimated from the mean velocity profiles using the analytic profile Musker (1979) based on the eddy viscosity model and given by the solution to the following implicit relation;

$$
\frac{d u^{+}}{d y^{+}}=\frac{\frac{y^{+2}}{k}+\frac{1}{s}}{y^{+3}+\frac{y^{+2}}{k}+\frac{1}{s}}
$$

where $s=0.001093$ according to Kendall and Koochesfahani (2008). They also stated that the Musker profile fits better experimental data than the logarithmic profile and, hence, potentially enhances the accuracy of $C_{f}$ determination. Figure 12 plots all data obtained in the range of flow velocities under investigation, including the results of repeated experiments conducted at different days. It is found that the measured $C_{f}$ follows closely the empirical formula $C_{f}=0.059 \mathrm{Re}_{x}^{-0.2}$ based on the power-law velocity profile assumption (Schlichting 1968) with the deviation being less than $4 \%$. In addition, Clauser equilibrium parameter

$G=\sqrt{\frac{2}{C_{f}}} \frac{H-1}{H}$

for the reference coating ( $H$ being the shape factor of the boundary layer) is practically constant over the insert and equal to 6.65 , which agrees with existing literature (see, e. $g$., 
Johansson and Alfredsson 1982) for equilibrium turbulent boundary layers. Deviation of measured $G$ values about mean appeared to be less than $\pm 5 \%$.

Due to the relatively weak amplitudes of pressure fluctuations at the wall in the velocity range under considerations, the expected interaction of the compliant coatings and the flow is rather small. Hence, the scatter of the data in Fig. 12 implies that the detection of possible skin friction drag reduction by a compliant coating is quite a delicate matter with the available tools. Hence, the hot-wire measurements were carried out with the reference coating and the compliant coating placed side by side, as depicted in Fig. 7 (a). The fiber-film probe was traversed between two coatings, making velocity profile measurements at one coating after the other at a fixed streamwise coordinate $x$ and a flow velocity. Then, the velocity was adjusted and the measurements repeated. After that, the probe was moved to another $x$ and the set of the measurements was repeated. Finally, new compliant coating was mounted and tested in the same manner, etc. Such a procedure reduced the scattering of the experimental data when comparing the difference.

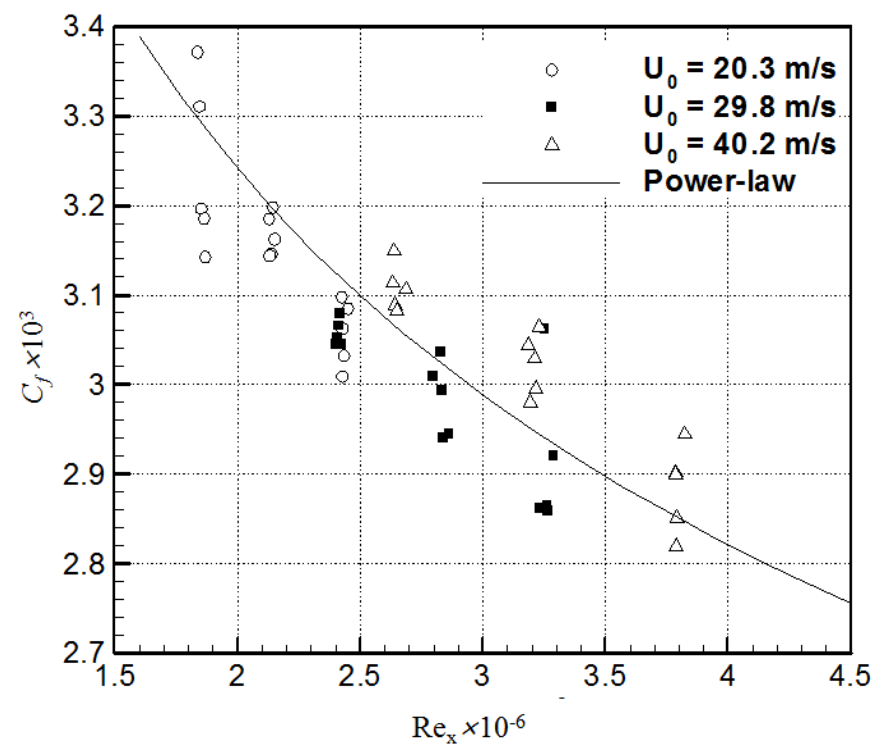

Fig. 12 Local skin friction coefficients at $U_{0}=20.3(\circ), 29.8$ $(\bullet), 40.2 \mathrm{~m} / \mathrm{s}(\Delta)$; correlation from power-law (solid line).

The skin friction reduction ratios $C_{f} / C_{f 0}=f\left(U_{0}\right)$ for four coatings under study are shown in Fig. 13. Here, $C_{f}$ and $C_{f 0}$ denote the skin frictional coefficients for the compliant coating and the reference coating, respectively. The values of $C_{f}$ were obtained using the Musker profile of Eq. (5) for both the reference coating and the compliant coatings. The region above the horizontal dotted lines $\left(C_{f} / C_{f 0}=1\right)$ corresponds to the area of increase of the local skin friction; correspondingly, the region below the horizontal lines corresponds to the area of the local skin friction reduction. It is seen that Fig. 13 indicates a clear tendency of skin friction reduction with increasing flow velocity. Among the coatings under study, C10.0 $7 \mathrm{~mm}$ exhibits in general the largest drag reduction of around $4 \%$ at $U_{0}=40.2 \mathrm{~m} / \mathrm{s}$ at all three downstream locations.

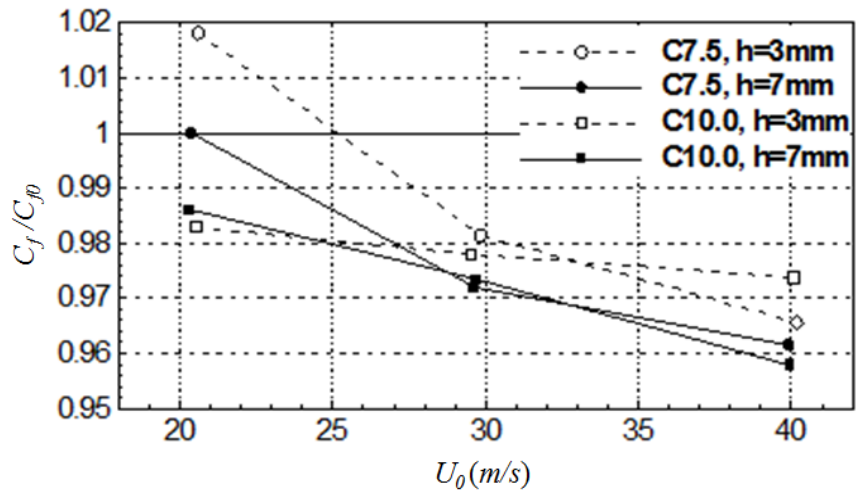

(a) $x=0.975 \mathrm{~m}$.

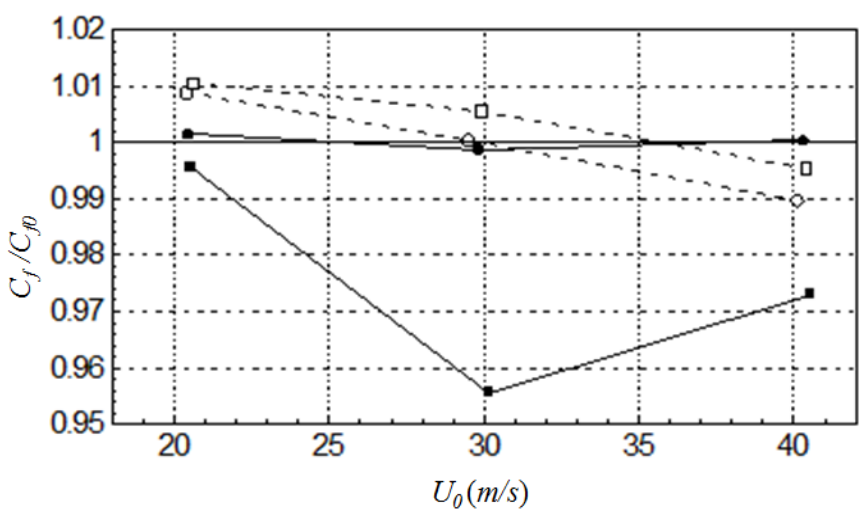

(b) $x=1.190 \mathrm{~m}$

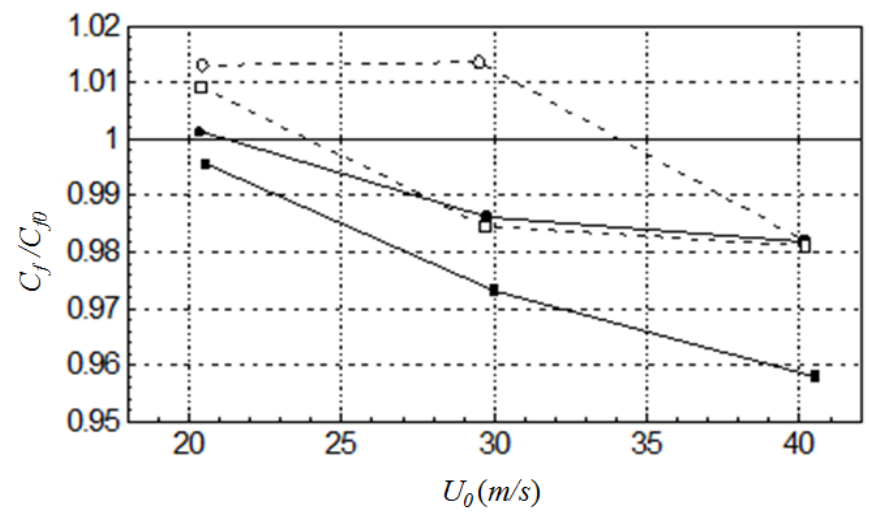

(c) $x=1.420 \mathrm{~m}$.

Fig. 13 Skin friction reduction ratio $C_{f} / C_{f 0}$.

Figure 14 gives the results of the balance measurements as the average skin friction coefficient defined as

$C_{F}=\frac{2 W}{\rho U_{0}^{2} S}$,

where $W$ is the frictional force measured by the strain gage balance and $S$ is the area of the insert. Since the insert 
extends in the steamwise range of $L_{1} \leq x \leq L_{2}, C_{f}$ could be correlated with the skin frictional drag coefficient

$$
\bar{C}_{f}(L)=\frac{1}{L} \int_{0}^{L} C_{f}(x) d x
$$

defined for the plate of length $\mathrm{L}(0 \leq x \leq L)$ as

$$
C_{F}=\frac{L_{2} \bar{C}_{f}\left(L_{2}\right)-L_{1} \bar{C}_{f}\left(L_{1}\right)}{L_{2}-L_{1}} .
$$

In Fig. 14, measured data of $C_{f}$ for the three coatings (reference, C7.5 $h=7 \mathrm{~mm}, \mathrm{C} 10.0 \mathrm{~h}=7 \mathrm{~mm}$ ) are shown in comparison with a theoretical estimation of Eq. (6) based on the correlation

$$
\bar{C}_{f}(L)=\frac{0.455}{\left(\lg \operatorname{Re}_{L}\right)^{2.58}}
$$

by Schlichting (1968). It is observed that the skin friction drag is reduced $3.6 \sim 6.0 \%$ with respect to the reference coating as the flow velocity increases to $U_{0}=30 \sim 40 \mathrm{~m} / \mathrm{s}$, which is largely in accordance with the local skin friction reduction obtained from hot-wire measurement.

The relatively large uncertainty of the measurements is attributable to the sensitivity of the balance readings to temperature changes inside the wind tunnel at $U_{0} \approx 40 \mathrm{~m} / \mathrm{s}$.

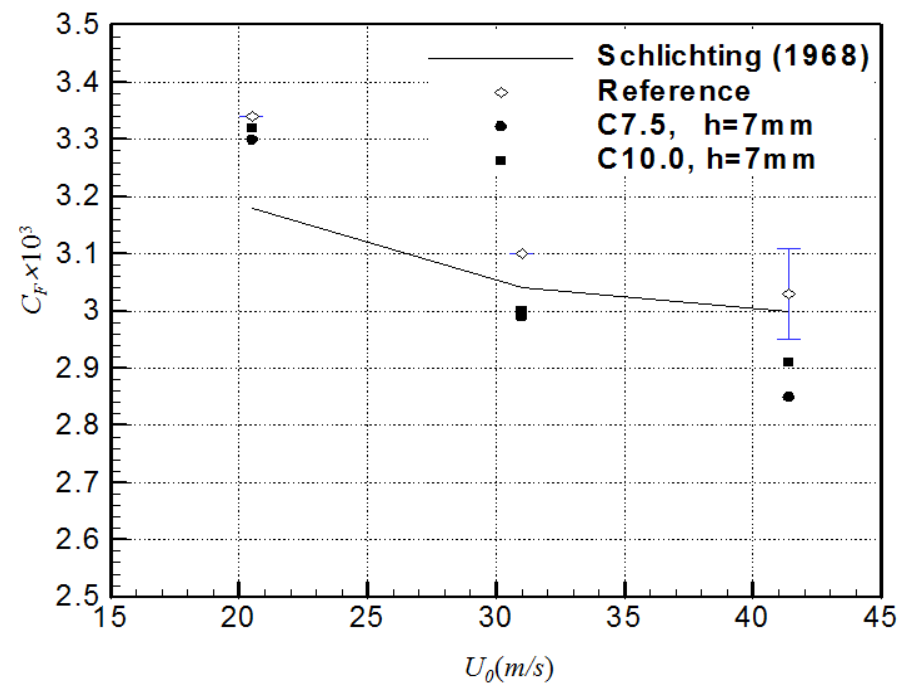

Fig. 14 Average skin friction coefficient from strain balance measurement.

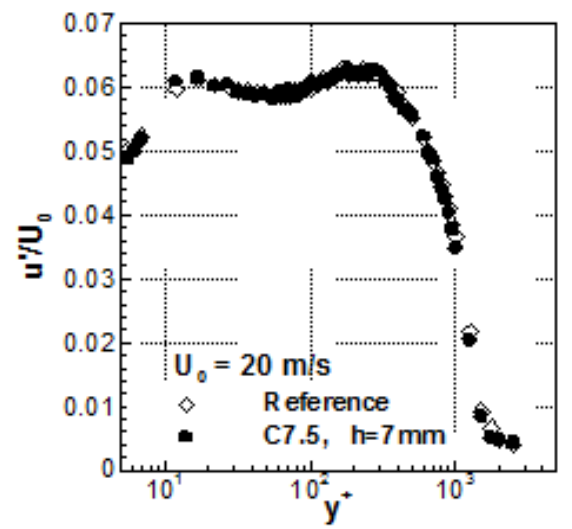

(a)

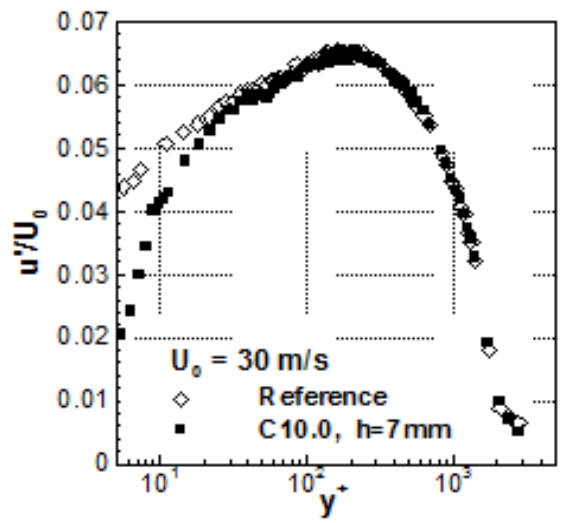

(d)

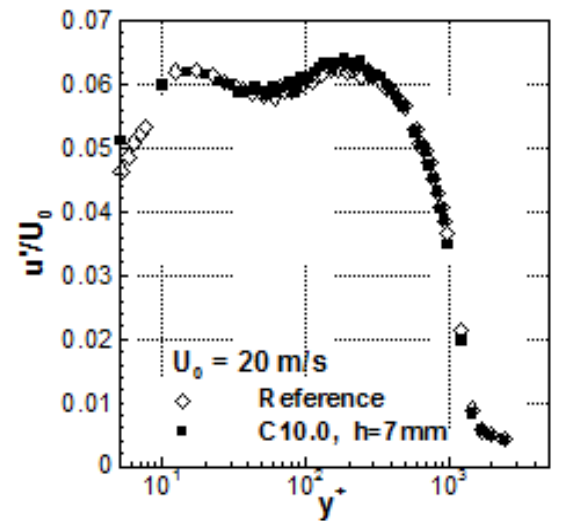

(b)

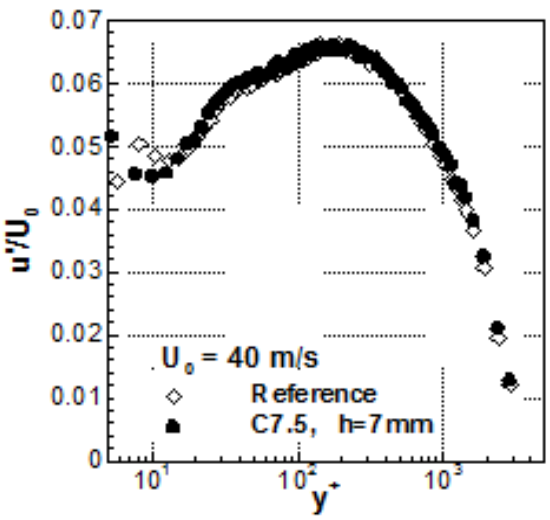

(e)

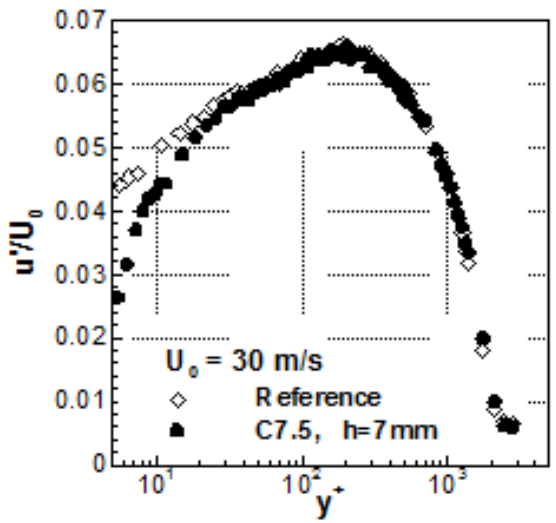

(c)

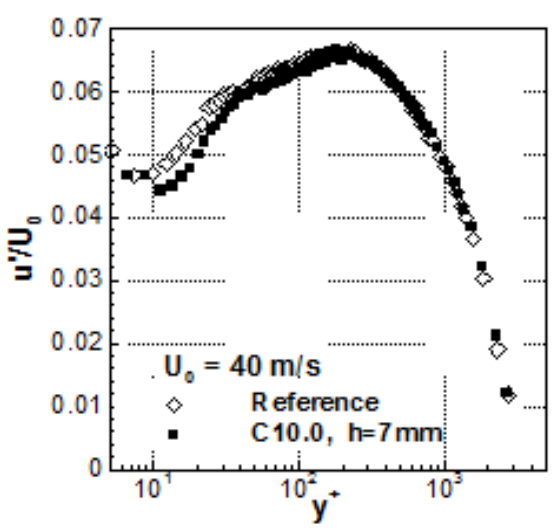

(f)

Fig. 15 Comparison of turbulence intensity profiles. 


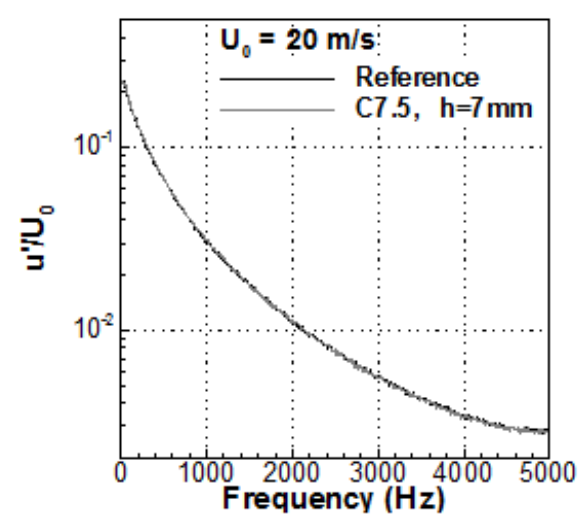

(a)

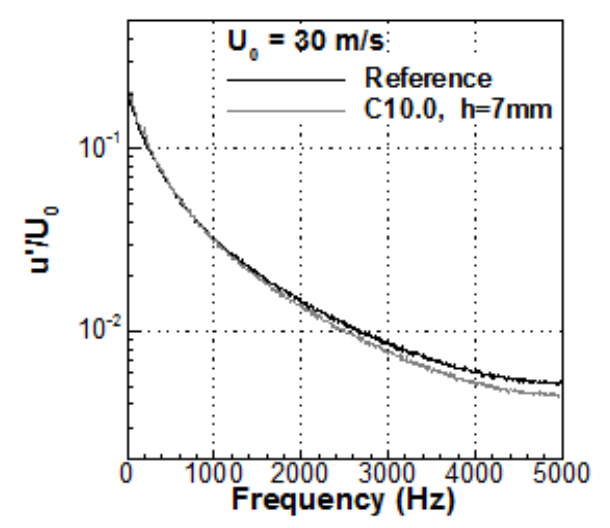

(d)

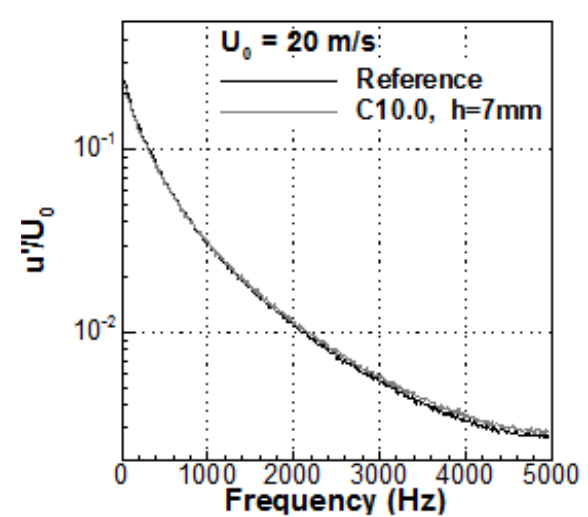

(b)

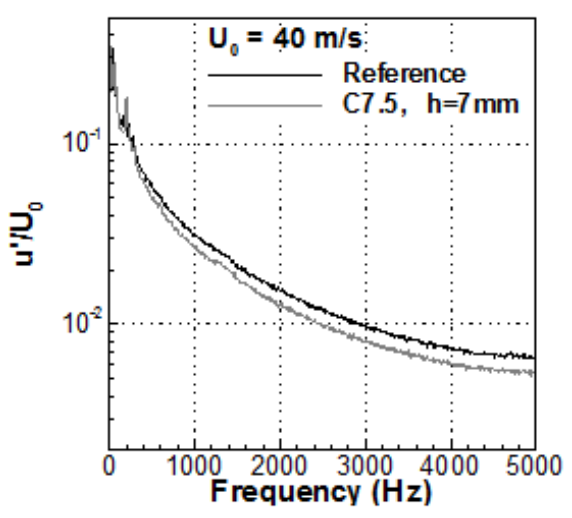

(e)

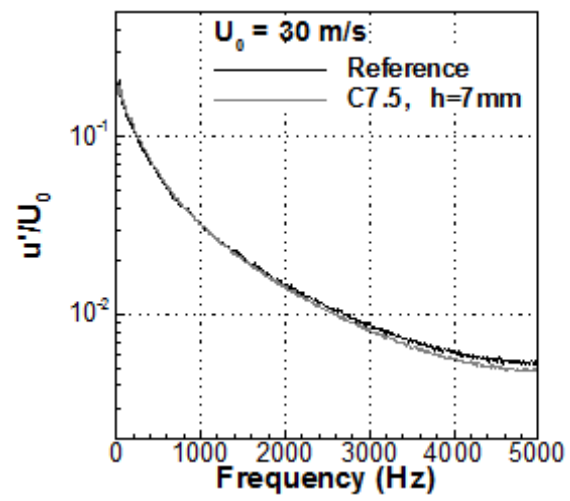

(c)

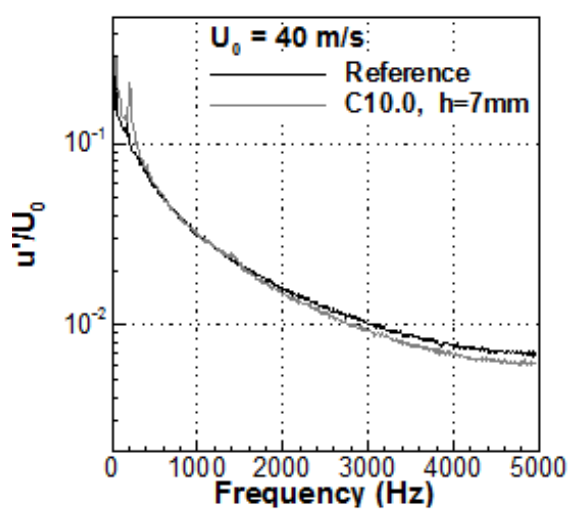

(f)

Fig. 16 Comparison of average spectra of velocity fluctuations in the near wall region $\left(y^{+} \leq 30\right)$.

The comparisons of profiles of rms velocity fluctuations between the reference cases and the respective coating cases are presented in Fig. 15. It is found that for the flow velocity $U_{0}=20 \mathrm{~m} / \mathrm{s}$ there is scarcely a notable difference between the profiles of velocity fluctuations, as in Figs. 15 (a) and (b). As the flow velocity becomes greater for $U_{0}=30 \mathrm{~m} / \mathrm{s}$ (Figs. 15 (c) and (d)), however, the profiles with compliant coating exhibit the significant attenuation of turbulent energy in the near wall region $y^{+} \leq 30$. This corroborates the basic idea of the present compliant coating design, which aims at a turbulent drag reduction associated with the attenuation of the turbulent velocity fluctuations. Considering that the optimum flow velocity obtained in Table 2 by overlapping the spatial and the temporal factors is near $U_{0}=30 \mathrm{~m} / \mathrm{s}$, the maximized attenuation of near wall velocity fluctuations for $U_{0}=30 \mathrm{~m} / \mathrm{s}$ is not considered to be entirely coincidental.

Figure 16 shows the turbulence spectrum for each compliant coating case in comparison with that for the corresponding reference case. The respective spectrum in Fig. 16 is not obtained from a single-point velocity measurement, but from the average of spectra measured in the near wall region $y^{+} \leq 30$. This was intended to avoid a misleading comparison of spectra measured at different wall normal distances. As a matter of fact, the comparison of individual spectra between a compliant coating case and a reference case at the same dimensional distances from the wall did not show a consistent tendency, i.e., sometimes reference spectrum spectra being smaller than compliant coating spectrum. However, the averaged spectra in Fig. 16 demonstrate that the present compliant coating gives rise to a significant attenuation of turbulent velocity fluctuations in a wide frequency ranges.

\section{CONCLUSIONS}

An experimental verification of a priori estimation of the efficiency of viscoelastic materials to reduce the skin frictional drag in turbulent boundary layer was performed for a set of stiff compliant coatings at moderate velocities of air flow typical for many commercial vehicles $(20.3 \sim 40.2 \mathrm{~m} / \mathrm{s}$, equivalent to about $70 \sim 140 \mathrm{~km} / \mathrm{h}$ ). The results demonstrated that the compliant coatings provided up to $4-5 \%$ (at $U_{0}=$ $30 \sim 40 \mathrm{~m} / \mathrm{s}, h=7 \mathrm{~mm}, E \approx 1.1 \mathrm{MPa}$ ) skin frictional drag reduction quite repeatedly, which is in qualitative accordance with the prediction. The velocity fluctuations profiles and spectra exemplified the mechanism of drag reduction, i.e., the attenuation of turbulence in the near wall region $\mathrm{y}^{+} \leq 30$.

For further verification of the method, it could be desirable to test its validity at higher velocities, e.g., for highspeed trains $(40 \sim 100 \mathrm{~m} / \mathrm{s}$ or about $140 \sim 360 \mathrm{~km} / \mathrm{h})$. Owing to the increased amplitudes of pressure fluctuations at the wall, 
more intensive interaction between the compliant coating (with properly tuned characteristics) and the flow would be obtained, thereby leading to more pronounced skin frictional drag reduction effect.

\section{ACKNOWLEDGEMENTS}

This research was supported by the GCRC program (GCRC-SOP; Global Core Research Center for Ships and Offshore Plants) of MEST/NRF of Korea.

\section{REFERENCES}

Amphilokhiev, V.B. Artyushkov, L.S. Barbanel, B.A. Korotkin, A.I. Mazaev, K.M. Maltsev, L.I. and Semenov, B.N., 2000. Modern State of the Theory of Boundary Layer Control. Saint-Petersburg: SUE "SPMDB Malachite"

Bandyopadhyay, P.R. Henoch, C. Hrubes, J.D. Semenov, B. N. Amirov, A.I. Malyuga, A.G. Choi, K-S. and Escudier, M.P., 2005. Experiments on the effects of ageing on compliant coating drag reduction. Phys. Fluids , 7(8), 085104.

Boiko, A.V. Kulik, V.M. Seoudi, B. Chun, H.H. and Lee, I., 2010. Measurement method of complex viscoelastic material properties. Int. J. of Solids and Structures, 45, (3-4), pp.374-382.

Bushnell, D.M. Hefner, J.N. and Ash, R.L., 1977. Effect of compliant wall motion on turbulent boundary layers. Phys. Fluids, vol. 20, pp.S31-S48

Carpenter, P.W., 1990. Status of transition delay using compliant walls. In: Viscous Drag Reduction in Boundary Layers, eds. D.M. Bushnell and J. N. Heffner, Progress in Astronautics and Aeronautics, 123, pp.79-113.

Carpenter, P.W. and Garrad, A.D., 1985. The hydrodynamic stability of flows over Kramer-type compliant surfaces. Part1. Tolmien-Schlichting instabilities. J. Fluid Mech., 155, pp.465-510.

Choi, K-S. Yang, X. Clayton, B.R. Glover, E.J. Atlar, M. Semenov, B.N. and Kulik, V.M., 1997. Turbulent drag reduction using compliant surfaces. Proc. Royal Soc. London A, 453, pp.2229-2240.

Duncan, J.H., Waxman, A M. and Tulin, M.P., 1985. The dynamics of waves at the interface between a viscoelastic coating and fluid flow. J. Fluid Mech., 158, pp.177-197.

Gad-el-Hak, M., 1996. Compliant coatings: a decade of progress, Appl. Mech. Rev., 49(10), part 2, pp.S147-S157

Gad-el-Hak, M. Blackwelder, R.F. and Riley, J.J., 1984. On the interaction of compliant coatings with boundary layer flows. J. Fluid Mech., 140, pp.257-280.
Huang, J-C. and Johnson, M.W., 2007. The influence of compliant surfaces on bypass transition. Exp. Fluids, 42 , pp.711-718.

Johansson, A.V. and Alfredsson, P.H., 1982. On the structure of turbulent channel flow. J. Fluid Mech., 122, pp.295314.

Kendall, A. and Koochesfahani, M., 2008. A method for estimating wall friction in turbulent wall-bounded flows. Exp. Fluids, 44, pp.773-780.

Kornilov, V.I. Pavlyuchenko, A. M. and Sobolev, A.V., 2004. Effect of a viscoelastic coating on friction drag of a flat plate in air flow. Thermophysics and Aeromechanics, 11(3), pp.405-415.

Kramer, M.O., 1957. Boundary layer stabilization by distributed damping. J. Aero. Sci., 24(6), pp.459-460.

Kramer, M.O., 1962. Boundary layer stabilization by distributed damping. Naval Engineers J., 74(2), pp.341348.

Kulik, V.M. Poguda, I.S. and Semenov, B.N., 1991. Experimental investigation of one-layer viscoelastic coating action on turbulent friction and wall pressure fluctuations. In: Recent Developments in Turbulence Management, ed. K.-S. Choi, Kluwer, pp.263-289

Kulik, V.M. Rodyakn, S.V. Lee, I. and Chun, H.H., 2005 a. Deformation of a viscoelastic coating under the action of convective pressure fluctuations. Exp. Fluids, 38(5), pp.648-655.

Kulik, V.M. Rodyakn, S.V. Suh, S-B. Lee, I. and Chun, H.H., 2005b. The response of compliant coating to nonstationary disturbances, Phys. Fluids, 17(8) 088104.

Kulik, V.M. Lee, I. and Chun, H.H., 2008. Wave properties of coating for skin friction reduction. Phys. Fluids, 20(7), 075109

Kulik, V.M. Semenov, B.N. Boiko, A.V. Seoudi, B. Chun, H. H. and Lee, I., 2009. Measurement of dynamic properties of viscoelastic materials. Exp. Mech., 49, pp.417-425.

Musker, A.J., 1979. Explicit expression for the smooth wall velocity distribution in a turbulent boundary layer. AIAA $J ., 17$, pp.655-657.

Schlichting, H., 1968. Boundary Layer Theory. McGraw-Hill, New York.

Semenov, B.N., 1991. On conditions of modeling and choice of viscoelastic coatings for drag reduction. In: Recent Developments in Turbulence Management, edited by K.S. Choi, Kluwer, pp.241-262.

Semenov, B.N., 1996. Analysis of four types of viscoelastic coating for turbulent drag reduction. Emerging Techniques in Drag Reduction, MEP, London, Edmunds

Semenov, B.N., 2009. Strategy of a choice of single-layer compliant coatings for turbulent drag reduction. Thermophysics and Aeromechanics, 16(2), pp.219-228. 